\title{
Mealtime assistants pilot project
}

\author{
R. Payne ${ }^{1}$, S. De Wet ${ }^{2}$, K. Sutton ${ }^{3}$ and H. Warwick ${ }^{1}$ \\ ${ }^{1}$ Department of Nutrition and Dietetics, Southampton General Hospital, Tremona Road, Southampton SO16 6YD, UK, \\ ${ }^{2}$ Speech and Language Therapy Department, Southampton General Hospital, Tremona Road, Southampton SO16 6YD, UK \\ and ${ }^{3}$ Volunteer Services, Southampton General Hospital, Tremona Road, Southampton SO16 6YD, UK
}

Elderly patients rarely meet their nutritional requirements while in hospital for a variety of reasons including a need for but lack of support $^{(1)}$. Using volunteers to support patients at mealtimes is a creative way of addressing this problem. A 3-year intervention is to start in Spring 2009 on the elderly care wards at Southampton General Hospital, where volunteers will be trained to support and feed vulnerable patients. Prior to this a 3-month pilot project was undertaken.

The aim of the pilot project was to investigate how having Mealtime Assistants (MTAs) would affect the calorie and protein intake of patients and provide an opportunity to trial the training programme, monitoring and support of MTAs. This would identify potential problems that might occur during the 3-year intervention.

A Senior Dietitian and Speech and Language Therapist (SLT) provided a practical half-day training session to 7 volunteers in October 2008. The session included safe feeding practices, looking for signs of difficulty when feeding patients and the nutritional needs of elderly care patients. The pilot ran for 3 months, with patients being fed at 2 lunchtimes each week, with 3 volunteers per mealtime. Nursing staff were responsible for identifying appropriate patients for volunteers to assist. Food charts were kept for 3 weeks, capturing days when the MTAs were on the ward and when no MTAs were available. On completion of the pilot the Dietitian, SLT, volunteers and ward staff were given an opportunity to feedback their comments.

Of the 80 patients identified, $88 \%$ (70 patients) were supported and/or fed by MTAs. Three weeks of food charts were analysed by the Dietitian for energy and protein intake. Change in calories ranged from a decrease of $25 \mathrm{kcal}$ to an increase of $438 \mathrm{kcal}$ when MTAs were present, giving an average increase of $187 \mathrm{kcal}(22 \%)$. Change in protein intake ranged from a decrease of $1.6 \mathrm{~g}$ to an increase of $11.5 \mathrm{~g}$ when MTAs were present, giving an average increase of $4.36 \mathrm{~g}$ protein $(37 \%)$.

The feedback from all staff and volunteers involved was very positive. The training and actual feeding of the patients were deemed the most positive aspects of the pilot. A number of issues were raised as things that 'could have gone better'. This included better information for volunteers around staff uniforms and job roles, working with nursing staff to improve their awareness of the MTAs role and the appropriateness of referrals to MTAs. It was also suggested that MTAs wear a uniform or tabard top to improve identification. There is also a need to formerly assess patients' and relatives' views of MTAs.

We have demonstrated that volunteers can be trained to safely feed and support patients at mealtimes, significantly improving their nutritional intake. During this small pilot project the amount of time needed by the Dietitian and SLT to run and support the project was greatly underestimated and needs to be factored into the 3-year project if it is to be safe and successful. This pilot was an excellent example of multi-disciplinary working and will be extremely valuable when planning for the 3-year project.

1. Age Concern (2006) Hungry to be Heard.Age Concern, http://www.ageconcern.org.uk/AgeConcern/Documents/Hungry_to_be_heard_August_2006.pdf 